\title{
Miniature Toroidal Radio Frequency Ion Trap Mass Analyzer
}

\author{
Stephen A. Lammert, ${ }^{*}$ Alan A. Rockwood,* Miao Wang, \\ and Milton L. Lee \\ Department of Chemistry and Biochemistry, Brigham Young University, Provo, Utah, USA \\ Edgar D. Lee, Samuel E. Tolley, James R. Oliphant, Jeffrey L. Jones, \\ and Randall W. Waite \\ Palmar Technologies, Highland, Utah, USA
}

\begin{abstract}
A miniature ion trap mass analyzer is reported. The described analyzer is a $1 / 5$-scale version of a previously reported toroidal radio frequency (rf) ion trap mass analyzer. The toroidal ion trap operates with maximum rf trapping voltages about $1 \mathrm{kV}_{p-p}$ or less; however despite the reduced dimensions, it retains roughly the same ion trapping capacity as conventional 3D quadrupole ion traps. The curved geometry provides for a compact mass analyzer. Unit-mass resolved mass spectra for $n$-butylbenzene, xenon, and naphthalene are reported and preliminary sensitivity data are shown for naphthalene. The expected linear mass scale with rf amplitude scan is obtained when scanned using a conventional mass-selective instability scan mode combined with resonance ejection. (J Am Soc Mass Spectrom 2006, 17, 916-922) () 2006 American Society for Mass Spectrometry
\end{abstract}

$\mathrm{B}$ ecause of the inherent high sensitivity and selectivity of mass spectrometry (MS), many applications benefit from the continued development of portable MS systems [1] including threat detection (e.g., chemical and biological agents, explosives), forensic investigations, environmental on-site monitoring, and illicit drug detection/identification. Key to developing portable mass spectrometers is reduction in size, weight, and power consumption along with reduced support utility requirements and cost. In addition, several crucial components such as vacuum system pumps must be miniaturized and made more rugged to allow portability.

Ion trap (IT) mass analyzers [2], by virtue of their simplicity, are ideal candidates for miniaturization. They are inherently small, even as implemented commercially, and have only a few ion optic elements, which do not require highly precise alignment relative to other types of mass analyzers. Since they are trapping devices, multiple stages of mass spectrometry $\left(\mathrm{MS}^{\mathrm{n}}\right)$ can be performed in a single mass analyzer. The operating pressure for ion traps is higher than for other forms of mass spectrometry, allowing for less stringent pumping requirements. Furthermore, since the radio frequency

Published online May 12, 2006

Address reprint requests to Dr. S. A. Lammert, Brigham Young University, Department of Chemistry and Biochemistry, C267 BNSN, Provo, UT 84602,

USA. E-mail: slammerts@byu.edulammerts@adelphia.net

* Adjunct Research Faculty. (rf) trapping voltage is inversely proportional to the square of the analyzer radial dimension, a modest decrease in analyzer size results in a large reduction in operating voltage and, hence, lower power requirements. An added potential benefit of the reduced analyzer size is the shorter ion path length, which may ease the vacuum requirements even further. This is an especially important area as some of the most limiting aspects of MS miniaturization are not in the ion optic components, but rather in the vacuum and other support assemblies.

The ability to miniaturize ion trap mass spectrometers hinges on addressing the issues of space charge [3] and machining tolerance limits. Miniature ion traps with conventional ion trap geometries (i.e., hyperbolic surfaces) have been previously explored [4]. However, as devices become smaller, the machining tolerances play an increasingly significant role in trapping field defects. Simplifying the geometry to one that is more easily machined is one approach to addressing this problem. Cylindrical ion trap [5] mass analyzers have been explored as the simplified, straight lines of a cylinder are considerably easier to machine than hyperbolic surfaces, especially at small dimensions. Ion traps with cylindrical geometry [6, 7] have been miniaturized. When the geometry of the analyzer electrodes deviates significantly from the theoretical geometry, as is the case for cylindrical ion traps, corrections are needed to restore the trapping field potentials to their theoretical values. Modeling 
and simulation programs [8] have been used extensively in this undertaking.

The gains from reducing analyzer size (e.g., increased portability attributable to lower weight and smaller size, lower rf generator power, and relaxed vacuum requirements) are offset by a reduction in ion storage capacity [9]. Concomitant with this reduced capacity is an earlier onset of space charge conditions, based on ion-ion repulsion, which results in reduced mass resolution and mass peak shifts. Efforts to address this constraint in ion trap mass spectrometers have lead to several approaches. Arraying several reduced volume cylindrical ion traps [10-12] is one approach to recovering the lost ion capacity. More recently, linear ion traps [13] with either radial $[14,15]$ or axial ejection [16] have been developed. The increased ion storage capacity is attributable to the volume available throughout the length of the two-dimensional quadrupole rod assembly. These devices are now readily available in commercial versions. For reasons similar to those where cylindrical ion trap geometries are used to approximate the 3D quadrupolar ion trapping field, a rectilinear ion trap [17] has been reported that uses a rectangular rod assembly instead of the more conventional hyperbolic quadrupole rod surface. All of these linear devices provide an increase in ion storage capacity by employing a traditional 2D quadrupole with ion gates on either end of the quadrupole rod assembly. In a few cases, these devices have been miniaturized [18] to sizes smaller than typical commercial linear quadrupoles although, to date, none have been miniaturized for the specific purpose of field portable instrumentation. Arrays of linear quadrupoles [19, 20] have also been reported.

Another analyzer geometry that offers increased ion storage and is amenable to miniaturization is the toroidal $\mathrm{rf}$ ion trap [21]. The device can be viewed as either a conventional 3D ion trap cross section that has been rotated on an edge through space or as a linear quadrupole curved and connected end to end. In either case, distortions to the quadrupole trapping field introduced by the curvature of the storage region degrade the performance of the device, and necessarily require corrections to the shape of the electrodes to generate the necessary trapping field. The result of the field corrections is a first-order, 2D quadrupole trapping field with a slight nonlinear (primarily octapole) field contribution intentionally added [22] to improve the ion ejection characteristics. Because of the geometry, the toroidal rf ion trap stores ions in a large-volume by distributing them within a circular storage ring. A miniature ion trap mass analyzer based on the previously reported toroidal rf ion trap geometry has been fabricated and preliminary data have been obtained.

The miniature analyzer employs the same optimized geometry (asymmetrically shaped electrodes [21] as its larger predecessor. Because of the reduced radial dimension $\left(\mathrm{r}_{0}\right)$ of the device, it operates at a considerably lower rf voltage $(\mathrm{V})$ as can be seen from the inverse relationship between the two variables in eq 1, which governs mass stability in a quadrupole ion trap mass spectrometer.

$$
q=\frac{-8 * e^{*} V}{m^{*}\left(r_{0}^{2}+2 z_{0}^{2}\right) * \Omega^{2}}
$$

In eq 1, q represents one of the two Mathieu stability parameters, $\mathrm{z}_{0}$ is the axial dimension, $\mathrm{m}$ is the ion mass, and $\Omega$ is the rf frequency.

Despite the reduced radial dimension, the miniature toroidal rf ion trap has approximately the same ion storage volume as a full size, commercial ion trap mass spectrometer with a $1 \mathrm{~cm}$ radial dimension. However, instead of operating at $\mathrm{rf}$ voltages of ca. $15 \mathrm{kV}_{p-p}$, as in the case of commercial ion traps, this device operates at less than $1 \mathrm{~V}_{p-p}$.

The toroidal rf ion trap geometry offers some unique advantages as a miniature mass analyzer. As an ion trap, it retains all of the advantages discussed earlier (size, simplicity, pressure tolerance, $\mathrm{MS}^{\mathrm{n}}$, etc.). All ions are contained within a single trapping field so, unlike arrays, there is no concern from a machining standpoint in matching the individual ion traps in an array or in interfacing ion sources or detectors to ensure equal rates of ion production in, or sampling from, each cell of the array. Multiple rf drivers could be used to correct for the small machining differences that might be present in an array of ion traps, but from the perspective of developing field portable instruments, multiple rf circuits are undesirable. It is also acknowledged that multiple ion trap cells of different sizes have been used to simultaneously and selectively trap ions of different $\mathrm{m} / \mathrm{z}$, but if the goal is miniaturizing a full-scan mass spectrometer system, this approach is limited by the number of cells that can be arrayed. From a theoretical field geometry standpoint, the trapping field is homogenous throughout the entire trapping volume (i.e., there are no end effects). Because of the strong dependence of the analyzer performance on machining quality, it remains to be determined if this advantage can be fully realized as the performance of the miniature toroidal mass analyzer evolves. Finally, similar to radial ejection linear quadrupole traps, but in contrast to axial ejection linear quadrupole traps, all ions of a given mass-tocharge $(\mathrm{m} / \mathrm{z})$ are simultaneously ejected at the prescribed point in the scan independent of their position in the trapping field at the time.

\section{Experimental}

The toroid mass analyzer consists of three sections: the trapping region, the ionizer assembly, and the detector assembly. The trapping region is formed from four separate electrodes (two "endcap" and two "ring" electrodes-inner and outer) as can be seen in the photograph in Figure 1. The names of the four electrodes arise historically from their 3D trap origin and indicate their location in the assembly (i.e., the filament endcap is the 


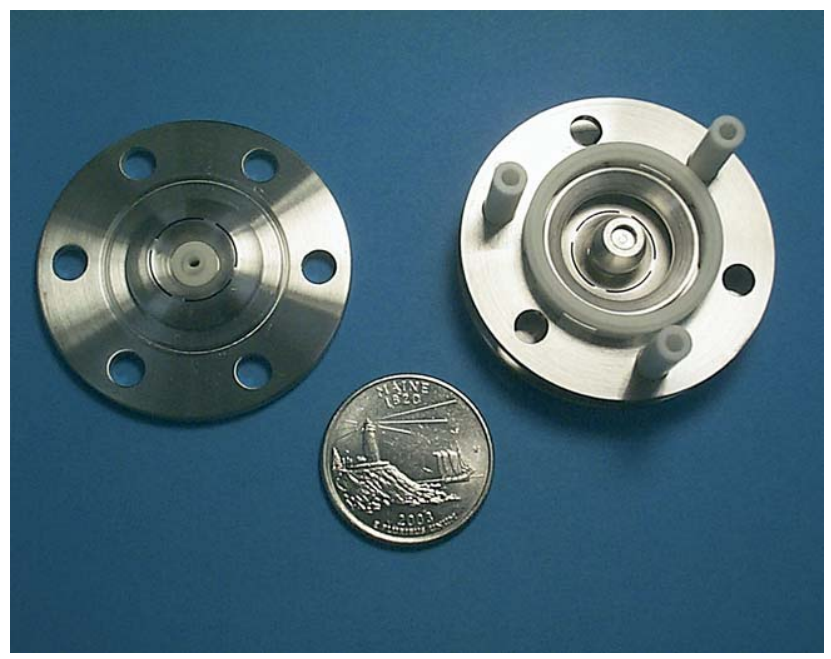

Figure 1. Photograph of the miniature toroidal rf ion trap analyzer showing the filament endcap (left) removed to expose the ion storage region (right). A U.S. quarter-dollar coin provides a size perspective.

electrode closest to the ionizer; the detector endcap is closest to the detector, etc.). While the traditional 3D ion trap "axial" and "radial" dimension terms would still be valid if only the cross-sectional trapping geometry is considered, a linear quadrupole model is seemingly more appropriate. For purposes of convention, the $\mathrm{x}$-dimension is defined here as the non-ejection dimension (towards the inner/outer rings), the y-dimension is defined as the ejection dimension (towards the endcaps), and the z-dimension is tangential to the toroidal trapping field. The electrodes were precision machined from 316 stainless steel with machine tolerances specified to 0.0005 inch $(0.013 \mathrm{~mm})$. The dimensions of the toroid assembly were scaled to $1 / 5$ of the size of the original version and retained the same shaped corrections to the trapping field [21] that were determined for the original version. The radius $\left(\mathrm{r}_{0}\right)$ of the miniature device is $0.2 \mathrm{~cm}$. Spacers were machined from alumina or Vespel and provide slits to allow gas conductance in and out of the trapping region. Entrance and exit slits with dimensions of $0.28 \mathrm{~mm}$ were machined into the two endcaps using a plunge electro-discharge machining (EDM) technique to allow the ionization electrons to enter into the trapping region and ejected ions to exit to the detector.

The entire mass analyzer consists of the above described toroidal rf ion trap, an electron impact (EI) ion source, and a detector. A cross-section schematic of this assembly is shown in Figure 2. A miniature electron gun was designed to focus source electrons, formed by emission from a heated filament, through a threeelement Einzel lens and then through a small section of the annular endcap slit. The middle lens (L2) of the electron focusing assembly also acted as a gate to turn the ionization on or off. A custom circuit was designed to switch the $\mathrm{L} 2$ voltage between $-50 \mathrm{~V}$ (ionization off) and $+125 \mathrm{~V}$ (ionization on). A current controlled power supply (Lambda, Model LPT-7202-FM, Plainview, NY) provided both the nominal $20 \mathrm{eV}$ electron energy and the nominal 1.5 A filament current.

Because of the smaller analyzer size, a conventional, more rugged continuous dynode electron multiplier detector (CDEM) was used in place of the microchannel plate detector previously used in the larger version. A custom CDEM (Detector Technologies, Palmer, MA) was developed to provide $>10^{5}$ gain at roughly $1 \mathrm{kV}$ for pressures up to $10^{-2} \mathrm{mbar}$. A custom circuit provided a gating voltage on a lens element between the detector endcap and the electron multiplier. This circuit switched between a maximum of $+50 \mathrm{~V}$ (detector gate closed) and $-50 \mathrm{~V}$ (detector gate open) to protect the detector from large ion currents during ionization. A high voltage power supply (Bertan/Spellman, Model 230, Hauppauge, NY) provided the detector voltage.

The rf trapping field was established using a custombuilt, class $C$ self-resonant tank circuit with a nominal frequency of $1.9 \mathrm{MHz}$. The rf signal was applied to both the inner and outer ring electrodes. The amplitude of the rf trapping voltage was typically ca. $100-200 \mathrm{~V}_{p-p}$ during the ionization and ion cooling portions of the scan. During mass analysis, the $\mathrm{rf}$ amplitude was then scanned over the course of $200 \mathrm{~ms}$ to ca. $700-1200 \mathrm{~V}_{p-p}$ depending on the mass range desired. At the end of each scan, the rf amplitude was turned off for a short (5 $\mathrm{ms}$ ) time period to allow all remaining ions to leave the trap before the next scan. An arbitrary waveform generator (Agilent, Model 33250A, Palo Alto, CA) was used to provide the resonant ejection [23] frequency $(900$ $\mathrm{kHz}, 8 \mathrm{~V}_{p-p}$ ) during the rf scan to produce the spectrum. This signal was applied to both endcaps through a custom Balun amplifier, which also contained a DC offset circuit to allow variations in the Mathieu " $a$ " parameter [9].

The entire mass spectrometer analyzer is only $5 \mathrm{~cm}$ in diameter and $10 \mathrm{~cm}$ long, including the ionizer and detector, which currently comprise the major portion of

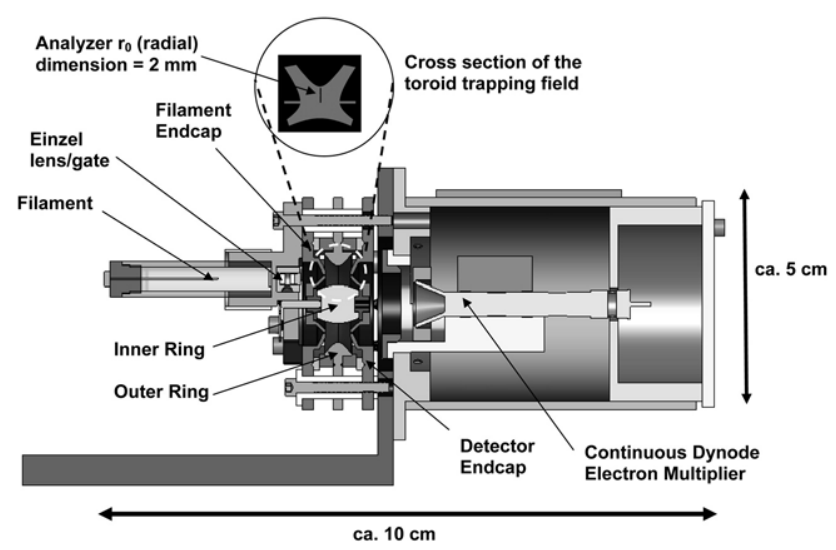

Figure 2. Cross-section schematic of the miniature toroidal rf ion trap analyzer showing the major components. The inset shows a cross-section of the miniature toroidal rf ion trap showing the shape corrections to the electrodes. The ion trap radius $\left(r_{0}\right)$ of 2.0 $\mathrm{mm}$ is highlighted in the inset. 


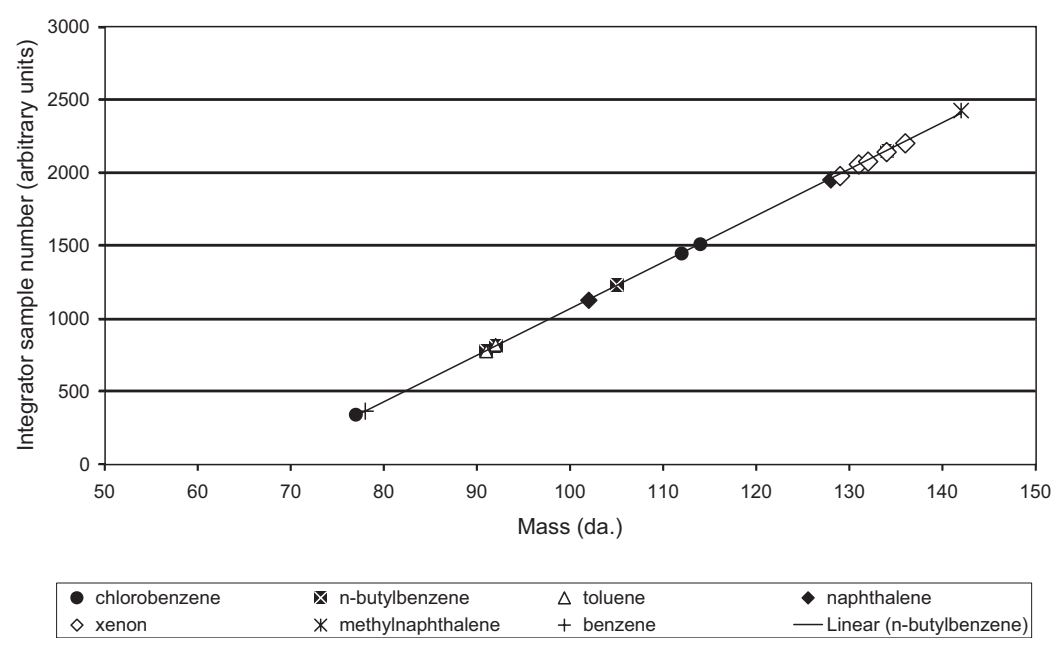

Figure 3. Mass calibration using seven compounds. Masses used for calibration from each compound are in parenthesis: chlorobenzene $[77,112,114]$; $n$-butylbenzene $[91,92,105,134]$; toluene [91, 92]; naphthalene [102, 128]; xenon [129, 131, 132, 134, 136]; methylnaphthalene [142]; benzene [78].

the length. It was enclosed in a custom vacuum chamber pumped by a $230 \mathrm{~L} / \mathrm{s}$ turbo pump (BOC Edwards, Model EXT255, Crawley, West Sussex, UK) backed by a $20 \mathrm{~m}^{3} / \mathrm{h}$ mechanical pump (BOC Edwards, Model E2M18, Crawley, West Sussex, UK). Pressures were monitored using a full range cold cathode vacuum gauge and controller (Pfeiffer, Model PKR 251/261, Asslar, Germany). All pressure readings are reported as uncorrected values.

Samples were introduced neat, directly into the vacuum chamber through either precision needle valves (Nupro/Swagelok, Solon, $\mathrm{OH}$ ) or variable leak valves (Granville-Phillips, Boulder, CO). All samples were reagent grade as supplied by the manufacturer. As is typical for most ion trap mass spectrometers, helium buffer gas is used to kinetically cool the ions before mass analysis. Helium was admitted using the same precision needle valves described above to a nominal pressure of $3 \times 10^{-4}$ mbar.

A simple, four-segment scan function was used to acquire data. A period of ionization (10-100 ms) was followed by a period of ion cooling (ca. 10-30 ms). The third segment of the scan function ramped the rf to perform the mass analysis, followed by a period of time (ca. $5 \mathrm{~ms}$ ) during which the rf was turned off and all ions were eliminated from the trap in preparation for the next scan. The timing of the scan function segments was controlled by a PIC-16C770 (Microchip Technology, Chandler, AZ) digital microcontroller. The PIC was programmable through a $\mathrm{I}^{2} \mathrm{C}$ interface. A Windows-based control program, Aardvark $\mathrm{I}^{2} \mathrm{C}$ Control Center (Total Phase, Sunnyvale, CA), was used to set the timing values in the PIC. The digital I/O outputs from the PIC were then used to control the initiation of the signal acquisition integrator, the start of the rf ramp, the control of the arbitrary waveform generator, the gating of the ionizer and detector, and the rf on/off status.
A multifunction data acquisition (DAQ) board (National Instruments Model 6115, Austin, TX) coupled with a multiport connector panel (National Instruments, Model BNC 2110, Austin, TX) was used to provide a variable modulation voltage to the rf generator to control the rf ramp. Signal from the detector was integrated using a custom preamplifier and sent to either a digital oscilloscope (Agilent, Model 64522A, Palo Alto, CA) or to the DAQ board where it was digitized by one of the analog-to-digital channels of the multifunction DAQ. A synchronization pulse started both the signal integrator and the rf ramp simultaneously. The signal integrator operated at $30 \mathrm{kHz}$ and provided digitized signal intensity every $33.3 \mu \mathrm{s}$. A typical rf scan would cover $\sim 300 \mathrm{Da}$ in $200 \mathrm{~ms}$, allowing $\sim 0.67 \mathrm{~ms}$ per dalton. Therefore at $30 \mathrm{kHz}$, there were $\sim 20$ samples acquired across each nominal mass. The number of signal integrations in the output was linear with time and, therefore, the signal integrator count was used to calibrate the mass scale for the acquired data. Operator input and data output were controlled and displayed on a custom instrument control screen programmed in Labview 7.1 (National Instruments, Austin, TX). Data manipulation for display included spectral averaging (typically 4-8 scans), Hanning filtering, and shot-noise rejection. Since the current Labview instrument control and acquisition program is rudimentary, all spectra shown are screen captures of the spectra as displayed on the user interface.

\section{Results and Discussion}

In the early stages of development, a resonant ejection frequency scan [24] was employed for mass analysis. With this approach, a range of frequencies was swept (from high-frequency to low-frequency) to eject ions held in the trap at a constant rf trapping level. Early on, it was believed that by using this scheme, a simpler scan 
(a)

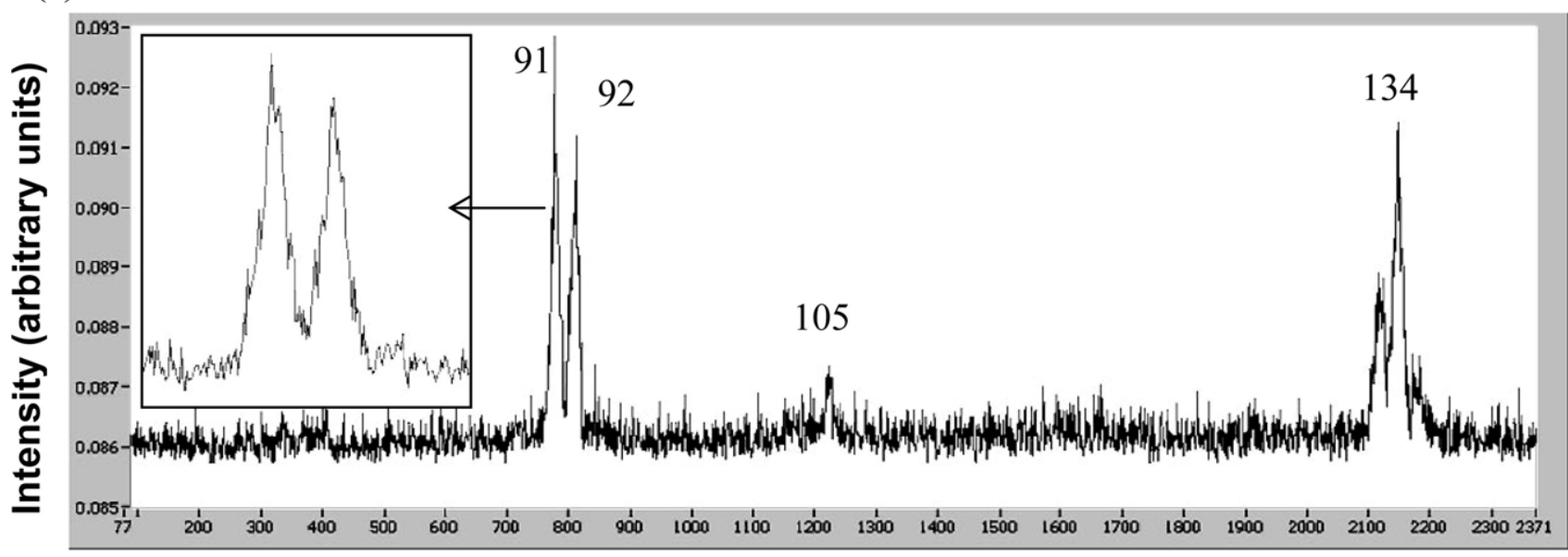

Integrator Sample Number (arbitrary units)

(b)

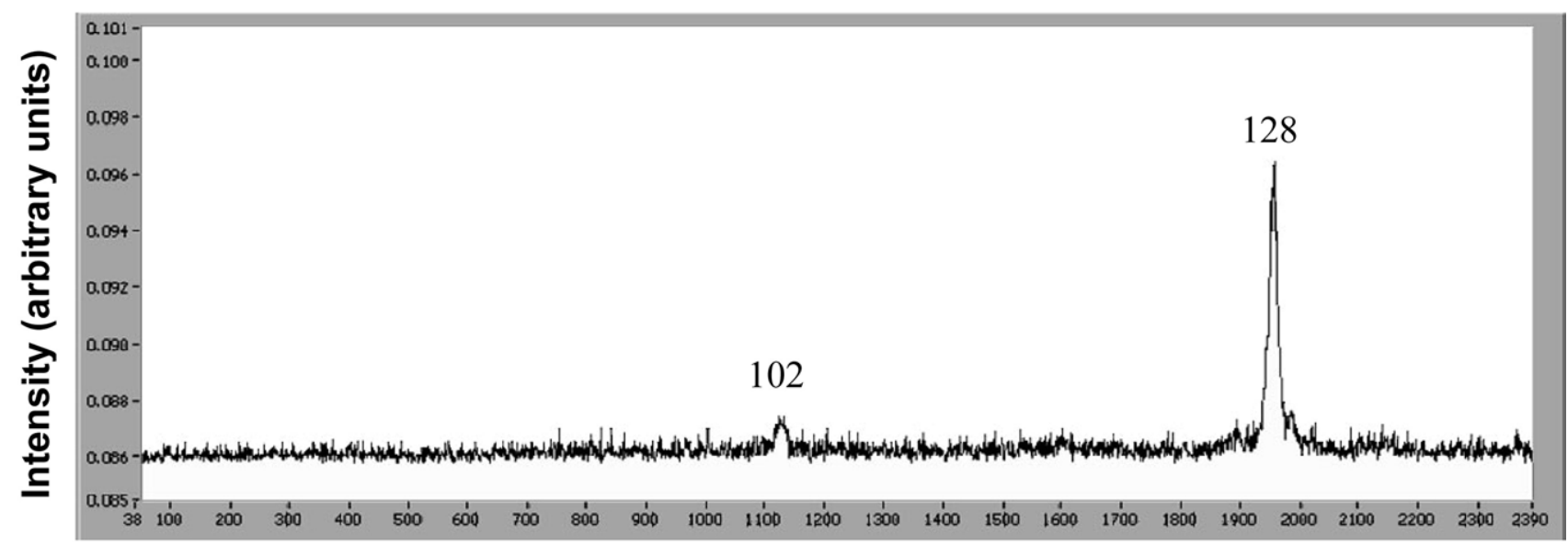

Integrator Sample Number (arbitrary units)

Figure 4. (a) Spectrum acquired for $n$-butylbenzene at a sample pressure of $1 \times 10^{-5}$ mbar. Full-width, half-maximum mass resolution for the $\mathrm{m} / \mathrm{z} 91$ peak is 0.4 da. Peaks are assigned with nominal $\mathrm{m} / \mathrm{z}$ values derived from a calibration table. (b) Spectrum acquired for naphthalene at low sample pressure $\left(2 \times 10^{-6} \mathrm{mbar}\right)$ and short ionization time $(20 \mathrm{~ms})$. The minor peak at $\mathrm{m} / \mathrm{z} 102$ in the naphthalene spectrum was still observed under these low sample pressure conditions. Peaks are assigned with nominal $\mathrm{m} / \mathrm{z}$ values derived from a calibration table.

electronics package could be developed since the rf generator needed only to be a fixed amplitude power supply. Results using this technique were disappointing as the spectra produced were often complicated by the presence of anomalous peaks. In addition, a sudden mass resolution loss was observed when shortening the mass scan limits beyond a certain threshold. Furthermore, the relationship between mass and ejection frequency was not linear, as indicated by theory, which complicated the mass calibration.

Because of these experiences, the frequency scan approach was abandoned in favor of the traditional $\mathrm{rf}$ amplitude scan coupled with resonance ejection, which yielded better results. Further improvements in the mass resolution of observed mass spectra were obtained when a new rf generator was fabricated, which allowed the frequency of the fundamental rf trapping field to be raised from an early value of $1.3 \mathrm{MHz}$ to 1.9 $\mathrm{MHz}$.

Since the toroidal rf ion trap is a 2D trapping device, and if the field corrections employed to compensate for distortions introduced by the analyzer curvature are adequate, the cross-sectional trapping field should look very much like a linear quadrupole [25]. The implications of this are that, unlike traditional 3D ion traps, $\mathrm{q}$-values (and therefore $\beta$-values) for the $\mathrm{x}$ - and $\mathrm{y}$ dimensions should be the same. This requires, therefore, that the ion's secular frequency in the $x$ - and $\mathrm{y}$-dimensions is similar, if not the same. Because a significant contribution of higher order fields is expected to be present (introduced intentionally or unintentionally), coupling of the ion motion in the $x$ - and 
y-dimensions [26] is expected. Energy coupled into the ion's y-dimension motion during resonance ejection can subsequently be coupled into the $\mathrm{x}$-dimension motion, causing a spreading of the ion cloud during mass analysis. It was found that adding a small direct current (dc) voltage to either the endcaps or the ring electrode improved the mass resolution in the acquired data. A dc component alters the Mathieu "a" parameter in the stability equations. Because in a 2D quadrupole device, $\beta_{\mathrm{x}}=\beta_{\mathrm{y}}$ only for a-values equal to zero, any non-zero a-value will separate the $\beta_{\mathrm{x}}$ and $\beta_{\mathrm{y}}$ parameters (and correspondingly, separate the $\mathrm{x}$ and $\mathrm{y}$ secular frequencies). Typically, a negative voltage (ca. $-1 \mathrm{~V}$ ) was applied to both endcaps through the Balun box during operation to accomplish this.

Mass spectral data were obtained for seven different samples (benzene, $n$-butylbenzene, toluene, chlorobenzene, naphthalene, xenon, and methylnaphthalene) and used to create the mass calibration graph shown in Figure 3. Since the rf amplitude was scanned linearly and the sample integrator was initiated at the beginning of the rf ramp, there is a direct linear relationship between the sample integrator number and rf amplitude. The calibration table shows the expected linear relationship between mass and $\mathrm{rf}$ amplitude.

The spectrum obtained for $n$-butylbenzene (sample pressure $1 \times 10^{-5} \mathrm{mbar}$, uncorrected) is shown in Figure 4a. The inset shows the peak doublet at $\mathrm{m} / \mathrm{z}$ $91 / 92$, which is clearly resolved. Measurement of the mass resolution for the peak at $\mathrm{m} / \mathrm{z} 91$ yielded 0.4 da full-width, half-maximum (FWHM). The spectrum was obtained by scanning the rf amplitude from 190-770 $\mathrm{V}_{p-p}$ in $200 \mathrm{~ms}$. The rf frequency was $1.9 \mathrm{MHz}$ and a resonant ejection frequency of $900 \mathrm{KHz}$ (corresponding to an approximate $\beta_{\text {eject }}$-value of 0.95 ) was used to eject ions during the rf amplitude scan. A DC offset of -890 $\mathrm{mV}$ was applied to the endcaps (i.e., $\mathrm{a} \neq \neq 0$ ) during the rf scan. The scan rate of $200 \mathrm{~ms} / \mathrm{scan}$ is required to maintain unit mass resolution, but is slower than desired for effective capillary GC/MS performance. It remains undetermined if improvements to the analyzer design, operating conditions, and machining/assembly procedures can improve the mass resolution and thereby allow faster scan rates. Changes to the analyzer entrance and exit slit designs are already underway in an attempt to address this issue.

Currently, there is no quantitative sample introduction system (e.g., gas chromatograph) interfaced to the prototype system to determine limits of detection. Preliminary measurements were made, however, to assess the general system sensitivity. Despite the early stage of development, high signal-to-noise spectra were obtained for samples with pressures as low as $2 \times 10^{-6}$ mbar and ionization times as low as $20 \mathrm{~ms}$. The spectrum for naphthalene under these conditions is shown in Figure $4 \mathrm{~b}$. The minor peak in the naphthalene spectrum at $\mathrm{m} / \mathrm{z} 102$ is still clearly observable. This performance is on par with conventional 3D ion trap systems. Figure 5 shows the molecular ion region of the spec-

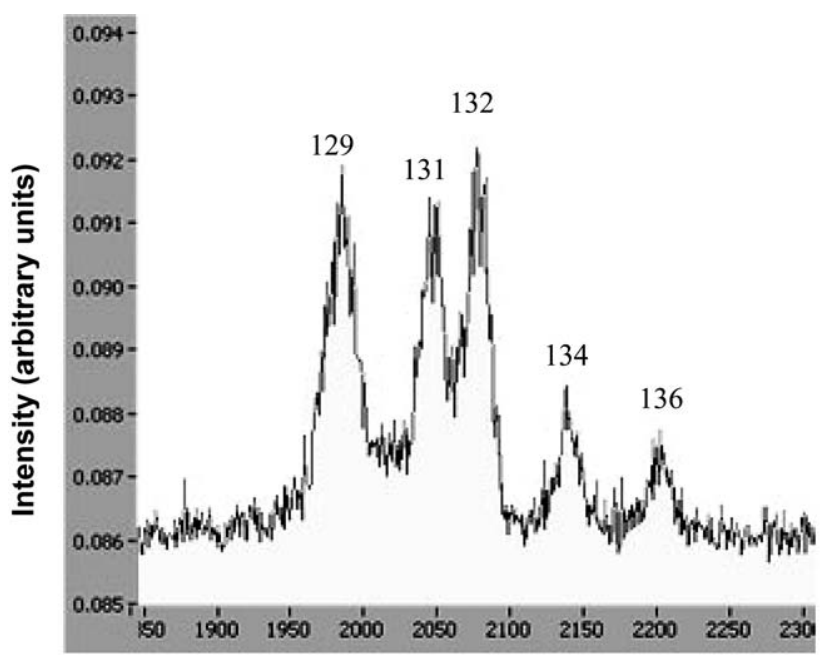

Integrator Sample Number (arbitrary units)

Figure 5. Spectrum acquired for xenon showing resolution of five xenon isotopes. Sample pressure was $2.3 \times 10^{-5}$ mbar. Peaks are assigned with nominal $\mathrm{m} / \mathrm{z}$ values derived from a calibration table.

trum obtained for xenon. The expected isotope pattern is observed and each of the isotopes is clearly resolved.

Further miniaturization of the toroidal rf ion trap mass analyzer is limited to a large degree by machining tolerances. As noted above, the electrode dimensions during machining were specified to 0.0005 in. tolerances, which are at or near the limits for current machining capabilities. The importance of tolerances in this device was highlighted during the development, when a prototype analyzer was improperly cleaned using a traditional aluminum oxide polishing method. The outer ring electrode shape was altered during the cleaning by $\sim 0.005 \mathrm{in}$. as measured by a coordinate measuring machine, which resulted in complete loss of ion signal when the system was reassembled and tested. Replacing the outer ring electrode restored normal system performance.

Improvements in mass resolution are possible as efforts continue to optimize operating parameters, including the $\mathrm{rf}$ drive frequency, $\beta_{\text {eject }}$-value, and the Mathieu a-value (from applying DC offset to the endcaps). In addition, further improvements in the rf amplifier (higher frequency, increased frequency, and amplitude stability) will also provide improved mass resolution, which may allow the currently slow scan speed to be increased without sacrificing unit mass resolution. As a prototype system, cabling and shielding is not yet optimum, leading to a higher than desired system electronic noise level. Trapping field simulation programs can be employed using electric field analysis programs such as Femlab (Comsol, Stockholm, Sweden) or POISSON (Los Alamos National Laboratory, Los Alamos, NM) to further refine the quality of the toroidal trapping field, especially the non-ejection (x) dimension. It is known from simulation studies on the fullsize version of the toroid ion trap that the non-ejection 
(x) dimension still has a significant non-linear character [21]. Previous efforts in geometry optimization on the full-size version focused only on one dimension of the trapping field, namely the ejection (y) dimension. Finally, efforts are already underway to develop a miniature mass spectrometer system with a small vacuum chamber, vacuum pumps, gas chromatograph, and electronics package.

\section{Conclusions}

Miniaturization of the toroidal rf ion trap mass analyzer has been achieved without sacrificing mass resolution or system sensitivity. Despite analyzer dimensions that approach the machining limits for a complex shape, unit mass resolution has been demonstrated. The relatively low rf power requirements and small analyzer components offer promise for the development of smaller, portable mass spectrometer systems.

\section{Acknowledgments}

This project was funded by the Department of Defense, Defense Threat Reduction Agency (DTRA, contract number DTRA 01-03C-0047 PZ0001) and Dugway Proving Ground (contract number DABJ 19-03-C-0005).

\section{References}

1. Badman, E. R.; Cooks, R. G. J. Miniature Mass Analyzers. Mass Spectrom. 2000, 35, 659-671.

2. March, R. E.; Todd, J. F. J., Eds.; Practical Aspects of Ion Trap Mass Spectrometry; Vols I-III; CRC Press: Boca Raton, FL, 1995.

3. Guan, S.; Marshall, A. G. Equilibrium Space Charge Distribution in a Quadrupole Ion Trap. J. Am. Soc. Mass Spectrom. 1994, 5, 64-71.

4. Kaiser, R. E.; Cooks, R. G.; Stafford, G. C.; Syka, J. E. P.; Hemberger, P. H. Operation of a Quadrupole Ion Trap Mass Spectrometer to Achieve High Mass/Charge Ratios. Int. J. Mass Spectrom. Ion Processes 1991, 106, 79.

5. Wells, J. M.; Badman, E. R.; Cooks, R. G. A Quadrupole Ion Trap with Cylindrical Geometry Operated in the Mass-Selective Instability Mode. Anal. Chem. 1998, 70, 438-444.
6. Kornienko, O.; Reilly, P. T. A.; Whitten, W. B.; Ramsey, J. M. Micro Ion Trap Mass Spectrometry. Rapid Commun. Mass Spectrom. 1999, 13 50-53.

7. Patterson, G. E.; Guymon, A. J.; Riter, L. S.; Everly, M.; Griep-Raming, J.; Laughlin, B. C.; Ouyang, Z.; Cooks, R. G. Miniature Cylindrical Ion Trap Mass Spectrometer. Anal. Chem. 2002, 74, 6145-6153.

8. Guangxiang, W.; Cooks, R. G.; Ouyang, Z. Geometry Optimization for the Cylindrical Ion Trap: Field Calculations, Simulations, and Experiments. Int. J. Mass Spectrom. 2005, 241, 119-132.

9. March, R. E.; Londry, F. A. Theory of Quadrupole Mass Spectrometry. InPractical Aspects of Ion Trap Mass Spectrometry; Vol I; Todd, J. F. J.; March, R. E., Eds.; CRC Press: Boca Raton, FL, 1995; Chap II.

10. Badman, E. R.; Cooks, R. G. Cylindrical Ion Trap Array with Mass Selection by Variation in Trap Dimensions. Anal. Chem. 2000, 72, 5079-5086.

11. Badman, E. R.; Cooks, R. G. A. Parallel Miniature Cylindrical Ion Trap Array. Anal. Chem. 2000, 72, 3291-3297.

12. Reilly, P. T. A.; Kornienko, O.; Whitten, W. B.; Ramsey, J. M. Microscale Ion Traps: Two-Dimensional Arrays. Proceedings of the 48th ASMS Conference on Mass Spectrometry and Allied Topics; Long Beach, CA, June, 2000.

13. Douglass, D. J.; Frank, A. J.; Mao, D. Linear Ion Traps in Mass Spectrometry. Mass Spectrom. Rev. 2005, 24, 1-29.

14. Schwartz, J. C.; Senko, M. W.; Syka, J. E. P. A Two-Dimensional Quadrupole Ion Trap Mass Spectrometer. J. Am. Soc. Mass Spectrom. 2002, 13, 659-669.

15. Bier,, M. E., Syka, J. E. P. U.S. Patent 1995, 5, 420, 425.

16. Hager, J. M. A New Linear Ion Trap Mass Spectrometer. Rapid Commun. Mass Spectrom. 2002, 16, 512-526.

17. Ouyang, Z.; Wu, G.; Song, Y.; Li, H.; Plass, W. R.; Cooks, R. G. Rectilinear Ion Trap: Concepts, Calculations, and Analytical Performance of a New Mass Analyzer. Anal. Chem. 2005, 76, 4595-4605.

18. Boumsellek, S.; Ferran, R. J. Tradeoffs in Miniature Quadrupole Designs. J. Am. Soc. Mass Spectrom. 2001, 12, 633-640.

19. Ferran, R. J.; Boumsellek, S. High-pressure Effects in Miniature Arrays of Quadrupole Analyzers for Residual Gas Analysis from $10^{-9}$ to $10^{-2}$ Torr. J. Vac. Sci. Technol. A 1996, 14, 1258-1265.

20. Orient, O. J.; Chutjian, A.; Garkanian, V. Miniature, High-Resolution Quadrupole Mass Spectrometer Array. Rev. Sci. Inst. 1997, 68, 1393 1397.

21. Lammert, S. A.; Plass, W. R.; Thompson, C. V.; Wise, M. B. Design, Optimization and Initial Performance of a Toroidal rf Ion Trap Mass Spectrometer. Int. J. Mass Spectrom. 2001, 212(1-3), 25-40.

22. Syka, J. E. P. 1995; Commercialization of the Quadrupole Ion Trap. Todd, J. F. J.; March, R. E., Eds.; In Practical Aspects of Ion Trap Mass Spectrometry; Vol I; CRC Press: Boca Raton, FL, Chap IV.

23. Tucker, D. B.; Hameister, C. H.; Bradshaw, S. C.; Hoekman, D. J.; Weber-Grabau, M. The Application of Novel Ion Trap Scan Modes for High Sensitivity GC/MS. Proceedings of the 36th ASMS Conference on Mass Spectrometry and Allied Topics; San Francisco, CA, June, 1988; p 628.

24. Goeringer, D. E.; McLuckey, S. A.; Glish, G. L. Enhancement of Mass Resolution in the Quadrupole Ion Trap Via Resonance Ejection; Proceedings of the 39th ASMS Conference on Mass Spectrometry and Allied Topics, Nashville, TN, 1991p 532.

25. Dawson, P. H., Ed.; Quadrupole Mass Spectrometry and Its Applications; Elsevier: Amsterdam, 1976

26. Franzen, J.; Gabling, R. H.; Schubert, M.; Wang, Y. 1995; Non-Linear Ion Traps. Todd, J. F. J.; March, R. E., Eds.; In Practical Aspects of Ion Trap Mass Spectrometry; Vol I; CRC Press: Boca Raton, FL, Chap III. 